\title{
Medications Associated With Geriatric Syndromes and Their Prevalence in Older Hospitalized Adults Discharged to Skilled Nursing Facilities
}

\author{
Avantika A. Saraf, MPH ${ }^{1,2}$, Alec W. Petersen, MD³ , Sandra F. Simmons, PhD 1,4,5, John F. Schnelle, PhD ${ }^{1,4,5}$, \\ Susan P. Bell, MBBS, MSCl 1,2,4, Sunil Kripalani, MD, MSc ${ }^{6,7}$, Amy P. Myers, PharmD ${ }^{8}$, Amanda S. Mixon, MD, MS, MSPH5,6,7, \\ Emily A. Long, BA, BS ${ }^{1}$, J. Mary Lou Jacobsen, BA ${ }^{1,5}$, Eduard E. Vasilevskis, MD, MPH'1,5,6*
}

\begin{abstract}
${ }^{1}$ Center for Quality Aging, Department of Medicine, Vanderbilt University Medical Center, Nashville, Tennessee; '2Division of Cardiovascular Medicine, Department of Medicine, Vanderbilt University Medical Center, Nashville, Tennessee; ${ }^{3}$ School of Medicine, Vanderbilt University, Nashville, Tennessee; ${ }^{4}$ Division of Geriatrics, Department of Medicine, Vanderbilt University Medical Center, Nashville, Tennessee; ${ }^{5}$ Geriatric Research, Education and Clinical Center, VA Tennessee Valley Healthcare System, Nashville, Tennessee; ${ }^{5}$ Section of Hospital Medicine, Division of General Internal Medicine and Public Health, Department of Medicine, Vanderbilt University Medical Center, Nashville, Tennessee; ${ }^{7}$ Center for Clinical Quality and Implementation Research, Vanderbilt University Medical Center, Nashville, Tennessee; ${ }^{8}$ Department of Pharmaceutical Services, Vanderbilt University Medical Center, Nashville, Tennessee.
\end{abstract}

BACKGROUND: More than half of the hospitalized older adults discharged to skilled nursing facilities (SNFs) have more than 3 geriatric syndromes. Pharmacotherapy may be contributing to geriatric syndromes in this population.

OBJECTIVES: Develop a list of medications associated with geriatric syndromes and describe their prevalence in patients discharged from acute care to SNFs.

DESIGN: Literature review and multidisciplinary expert panel discussion, followed by cross-sectional analysis.

SETTING: Academic medical center in the United States

PARTICIPANTS: One hundred fifty-four hospitalized Medicare beneficiaries discharged to SNFs.

MEASUREMENTS: Development of a list of medications that are associated with 6 geriatric syndromes. Prevalence of the medications associated with geriatric syndromes was examined in the hospital discharge sample.

RESULTS: A list of 513 medications was developed as potentially contributing to 6 geriatric syndromes: cognitive impairment, delirium, falls, reduced appetite or weight loss, urinary incontinence, and depression. Medications included 18 categories. Antiepileptics were associated with all syndromes, whereas antipsychotics, antidepressants, antiparkinsonism, and opioid agonists were associated with 5 geriatric syndromes. In the prevalence sample, patients were discharged to SNFs with an overall average of 14.0 ( \pm 4.7$)$ medications, including an average of 5.9 ( \pm 2.2$)$ medications that could contribute to geriatric syndromes, with falls having the most associated medications at discharge at $5.5( \pm 2.2)$.

CONCLUSIONS: Many commonly prescribed medications are associated with geriatric syndromes. Over $40 \%$ of all medications ordered upon discharge to SNFs were associated with geriatric syndromes and could be contributing to the high prevalence of geriatric syndromes experienced by this population. Journal of Hospital Medicine 2016;11:694700. (C) 2016 Society of Hospital Medicine
Geriatric syndromes are common clinical conditions in older adults that do not fall into specific disease categories. Unlike the traditional definition of a syndrome, geriatric syndrome refers to a condition that is mediated by multiple shared underlying risk factors. ${ }^{1,2}$ Conditions commonly referred to as geriatric syndromes include delirium, cognitive impairment, falls, unintentional weight loss, depressive symptoms, and incontinence. Even though many perceive it as medical misnomer, ${ }^{3}$ geriatric syndromes have been shown to negatively impact quality of life and activities of daily

\footnotetext{
*Address for correspondence and reprint requests: Eduard E. Vasilevskis, MD, 121521 st Avenue S, 6006 Medical Center East, NT, Nashville, TN 37232-8300; Telephone: 615-936-1935; Fax: 615-936-1269; E-mail: ed.vasilevskis@vanderbilt.edu

Additional Supporting Information may be found in the online version of this article.

Received: January 5, 2016; Revised: April 25, 2016; Accepted: April 28 2016

2016 Society of Hospital Medicine DOI 10.1002/jhm.2614

Published online in Wiley Online Library (Wileyonlinelibrary.com).
}

living in older adults. ${ }^{2}$ They are also associated with adverse outcomes such as increased healthcare utilization, functional decline, and mortality, even after adjusting for age and disease severity. ${ }^{4-6}$ Hospitalized older adults, including those discharged to skilled nursing facilities $(\mathrm{SNFs})^{7,8}$ are particularly at high risk for new-onset or exacerbation of geriatric syndromes and poor outcomes. ${ }^{7,9,10}$ However, hospital providers seldom assess, manage, or document geriatric syndromes because they are often overshadowed by disease conditions that lead to an acute episode requiring hospitalization (e.g., heart disease). ${ }^{11}$

Pharmacotherapy is the cornerstone of hospital treatment, and it is well-known that it affects multiple physiologic systems causing side effects apart from the condition they are approved to treat. Given that geriatric syndromes are a result of impairments in multiple organ systems, it is plausible that pharmacotherapy may initiate or worsen these syndromes. ${ }^{12}$ Medicationrelated problems in older adults are well known. Polypharmacy and adverse drug events (as a result of 
drug-drug/disease interactions and changes in pharmacokinetics and pharmacodynamics) are prevalent in multimorbid elderly patients. ${ }^{13-16}$ The prescribing cascade $^{17}$ increases the medication burden and may be a contributing factor for geriatric syndromes in hospitalized patients. ${ }^{18}$ For instance, laxatives may be prescribed to counteract constipation caused by anticholinergic drugs.

The American Geriatric Society (AGS) Beers list ${ }^{19,20}$ and similar criteria ${ }^{21}$ provide excellent resources to identify medications with potentially harmful interactions or adverse effects in older adults. Although these lists include medicines associated with a specific geriatric syndrome, they were not developed to explicitly link medicines across multiple geriatric syndromes, regardless of indication or appropriateness. For example, medications that effect important geriatric syndromes like unintentional weight/appetite loss, depression, and urinary incontinence are not extensively covered. In addition, disease-appropriate medications (eg, $\beta$-blockers for systolic heart failure), that may be associated with a geriatric syndrome (eg, falls) are not included; however, they may be important to consider for a patient and clinician who are weighing the disease benefits compared to the geriatric syndrome-related risks. Finally, the AGS 2015 Beers criteria panel mentions the limitation that many medication associations may be excluded because older adults are less represented in clinical trials. ${ }^{20}$ Clinicians are currently limited in identifying medications potentially contributing to a broad set of geriatric syndromes in their patients without a specific list of medications associated with geriatric syndromes (MAGS). ${ }^{20}$

In response to this gap, identifying these medications is important and should be a starting point in efforts toward prevention and treatment of geriatric syndromes. The 2 main objectives of this study were to first identify medications that may meaningfully contribute to 6 geriatric syndromes and subsequently describe the frequency of these medications in a population transitioning from acute care to post-acute care to highlight the need and potential impact of such a list.

\section{METHODS}

This study included 2 phases that aligned with our 2 primary objectives. Phase 1 involved identifying medications associated with 6 geriatric syndromes, and phase 2 included a cross-sectional analysis of the prevalence of these medications in a sample of patients discharged to SNFs.

\section{Phase 1: Development of the MAGS List}

Figure 1 depicts the underlying conceptual model and approach that was used in phase 1 . The interaction between the patient factors and medication leads to polypharmacy that contributes to geriatric syndromes and additional adverse outcomes. As a starting point for mitigating geriatric syndromes, we used an iterative analytical process to identify a list of medications associated with the following geriatric syndromes that were documented to be highly prevalent in patients discharged to SNFs: cognitive impairment, delirium, falls, unintentional weight and/or appetite loss, urinary incontinence, and depression. ${ }^{8}$ To be inclusive and sensitive, our approach differed from traditional systematic reviews, and in fact was meant to bring together much of the established systematic literature about disparate geriatric syndromes in 1 place, because patients often do not experience a geriatric syndrome in isolation, but rather experience multiple geriatric syndromes. ${ }^{8}$ The MAGS list had 3 main inclusion criteria (Figure 1): (1) evidence in the published literature (systematic reviews, cohort studies, randomized clinical trials) that the medication is related to the syndrome, (2) expert panel opinion, and (3) drug databases (Lexicomp Online database 22 and/ or US Food and Drug Administration [FDA]approved package inserts). ${ }^{23}$ We generated an initial list of medications based on these 3 main criteria to identify medications with significant associations to each geriatric syndrome. The list was further expanded and vetted using an iterative review of each medication list as it related to each geriatric syndrome through a series of group meetings focused around each geriatric syndrome. Following further discussion, we obtained agreement among all team members for medications included in the final list. For each geriatric syndrome, we excluded medications from consideration if they were used to treat the same geriatric syndrome (eg, $\alpha$-adrenergic blockers used to treat incontinence in men were listed as associated with incontinence only in women). We classified medications according to the Established Pharmacologic Class available at the FDA website. We also compared our final MAGS list with the 2015 AGS Beer's list ${ }^{20}$ by identifying medications that were related to the 6 geriatric syndromes. This included Beers ${ }^{20}$-cited rationale of anticholinergic, extrapyramidal symptoms, orthostatic hypotension (eg, falls), high-risk adverse central nervous system effects, sedating, cognitive decline (eg, antipsychotics), delirium, falls, fractures, incontinence, and gastrointestinal (eg, nausea, vomiting). Specifically, we assessed whether the medications were included as inappropriate by the AGS Beers $2015^{20}$ list and also whether they documented the syndrome association for that medication.

\section{Phase 2: Prevalence of MAGS in Hospitalized Older Adults Discharged to SNFs Sample}

We next applied the MAGS list to a convenience sample of hospitalized patients discharged to SNFs to assess the prevalence of MAGS in this sample, and also to compare with the prevalence of Beers criteria ${ }^{20}$ 

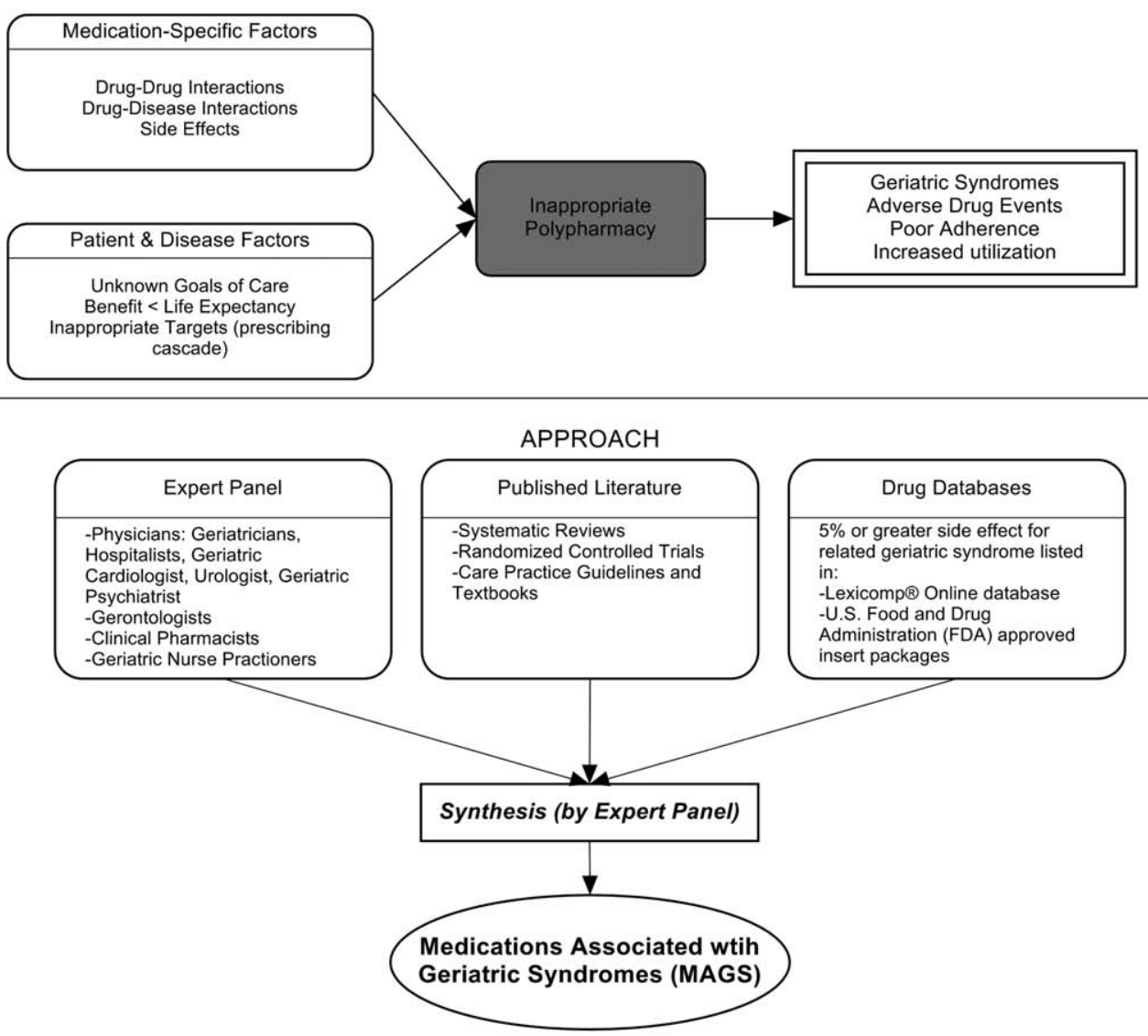

FIG. 1. Conceptual model and approach for development of the medication associated with geriatric syndromes (MAGS) list (phase 1).

medications. Our sample was selected from data collected as part of a quality-improvement project to reduce hospital readmissions in patients discharged to SNFs. The larger study enrolled a total 1093 medical and surgical patients who had Medicare insurance eligibility and were discharged from 1 large university hospital to 23 area SNFs from January 17, 2013 through July 31, 2014. The university institutional review board waived the requirement for written consent. For the purpose of this substudy. we selected the first 154 patients with complete chart abstraction (approximately $15 \%$ of the total) as a convenience sample.

\section{Data Analysis}

We applied descriptive statistics to summarize demographic and clinical characteristics of the convenience sample. To understand potential selection biases that could have resulted by the convenience sampling, we compared participant characteristics of the convenience sample $(\mathrm{N}=154)$ with the characteristics of the remaining participants of the larger study $(\mathrm{N}=939)$ using independent sample $t$ tests and $\chi^{2}$ tests for continuous and categorical measures, respectively. We applied the MAGS list and the AGS 2015 Beers criteria $^{20}$ for the sample of 154 and identified the medica- tions associated with each of the 6 geriatric syndromes from the discharge medication lists completed by hospital clinical pharmacists. For each patient, we identified both scheduled and PRN (pro re nata, or as needed) medications associated with each geriatric syndrome. Thereafter, we determined whether the discharge list contained at least 1 medication associated with a geriatric syndrome per the MAGS list and the AGS Beers 2015 criteria, ${ }^{20}$ and the percentage of overall medications that were part of the MAGS and Beers lists. Data were aggregated using means and standard deviations across syndromes (ie, number of discharge medications per syndrome per patient) along with the percentage of patients with 1 or more medications related to a specific syndrome and the percentage of medications that were MAGS. All analyses were performed using the SPSS statistical package (IBM SPSS Statistics for Windows, version 23.0; IBM, Armonk, NY).

\section{RESULTS}

\section{Phase 1: MAGS List}

The iterative process applied in this analysis generated a list of 513 medications associated with the 6 geriatric syndromes. The list of medications related to each syndrome and the corresponding rationale and 
TABLE 1. Summary of Medications Associated With Geriatric Syndromes

\begin{tabular}{|c|c|c|c|c|c|c|c|}
\hline $\begin{array}{l}\text { Major Medication } \\
\text { Category }\end{array}$ & Delirium & $\begin{array}{l}\text { Cognitive } \\
\text { Impairment }\end{array}$ & Falls & $\begin{array}{l}\text { Unintentional } \\
\text { Weight and } \\
\text { Appetite Loss }\end{array}$ & $\begin{array}{l}\text { Urinary } \\
\text { Incontinence }\end{array}$ & Depression & Drug Class/Drug Within Each Category \\
\hline Antipsychotics & & $\checkmark$ & $\checkmark$ & & $\checkmark$ & $\checkmark$ & Atypical and typical antipsychotics, buspirone \\
\hline Antidepressants & $\checkmark$ & $\checkmark$ & $\checkmark$ & $\checkmark$ & $\checkmark$ & & $\begin{array}{l}\text { Tricyclic and tetracyclic antidepressants, serotonin reuptake inhibitors, } \\
\text { serotonin and norepinephrine reuptake inhibitor, aminoketone }\end{array}$ \\
\hline Antiepileptics & $\checkmark$ & $\checkmark$ & $\checkmark$ & $\checkmark$ & $\checkmark$ & $\checkmark$ & Antiepileptics, mood stabilizers, barbiturates \\
\hline Antiparkinsonism & $\checkmark$ & $\checkmark$ & $\checkmark$ & & $\checkmark$ & $\checkmark$ & $\begin{array}{l}\text { Aromatic amino acid decarboxylation inhibitor and catechol-0-methyltransferase } \\
\text { inhibitor, catecholamine-depleting sympatholytic, catechol-0-methyltransferase } \\
\text { inhibitor, dopaminergic agonist, ergot derivative, monoamine oxidase inhibitor, } \\
\text { nonergot dopamine agonist, }\end{array}$ \\
\hline Benzodiazapines & $\checkmark$ & $\checkmark$ & $\checkmark$ & & & & Benzodiazapines only \\
\hline $\begin{array}{l}\text { Nonbenzodiazepine } \\
\text { hypnotics }\end{array}$ & $\checkmark$ & $\checkmark$ & $\checkmark$ & & & & $\begin{array}{l}\text { Benzodiazepine analogs, nonbenzodiazepine hypnotics, tranquilizers, } \\
\gamma \text {-aminobutyric acid A receptor agonist }\end{array}$ \\
\hline Opioid agonists & $\checkmark$ & $\checkmark$ & $\checkmark$ & & $\checkmark$ & $\sqrt{ }$ & Full or partial opioid agonists, opiates, opioids \\
\hline $\begin{array}{l}\text { Nonopioid/nonsteroidal } \\
\text { anti-inflammatory } \\
\text { and/or analgesics }\end{array}$ & & & $\checkmark$ & & & & Nonopioid analgesics, NSAIDS, COX-2 selective inhibitor NSAIDs \\
\hline Anthypertensives & & & $\checkmark$ & & $\checkmark$ & $\checkmark$ & $\begin{array}{l}\text { Calcium channel blocker, } \beta \text {-adrenergic blocker, angiotensin-converting enzyme inhibitor, } \\
\text { angiotensin } 2 \text { receptor blocker, } \alpha \text {-adrenergic blocker, diuretics } \\
\text { (loop, potassium sparing, thiazide), nitrate vasodilators, aldosterone blocker }\end{array}$ \\
\hline Antiarrhythmic & $\checkmark$ & $\checkmark$ & $\checkmark$ & & & & Antiarnythmics, cardiac glycosides \\
\hline Antidiabetics & & & $\checkmark$ & $\checkmark$ & & & $\begin{array}{l}\text { Insulin and insulin analogs, sulfonylureas, } \alpha \text {-glucosidase inhibitor, amylin analog, } \\
\text { biguanide, glinide, GLP-1 receptor agonist, glucagon-like peptide-1 agonist }\end{array}$ \\
\hline $\begin{array}{l}\text { Anticholinergics and/or } \\
\text { anthistaminics }\end{array}$ & $\checkmark$ & $\checkmark$ & $\checkmark$ & & $\checkmark$ & & $\begin{array}{l}\text { Anticholinergics, histamine receptor antagonists, muscarininc antagonists, combined } \\
\text { anticholinergics, and histamine receptor antagonists }\end{array}$ \\
\hline Antiemetics & $\checkmark$ & $\checkmark$ & $\checkmark$ & & & & Antiemetics, dopaminergic antagonists, dopamine-2 receptor antagonist \\
\hline Hormone replacement & & & & & & $\checkmark$ & $\begin{array}{l}\text { Corticosteroids, progestin, estrogen, estrogen agonist/antagonist, gonadotropin } \\
\text { releasing hormone receptor agonist }\end{array}$ \\
\hline Muscle relaxers & $\checkmark$ & d & $\checkmark$ & & $\checkmark$ & & Muscle relaxers \\
\hline Immunosuppressants & & & $\checkmark$ & & & & $\begin{array}{l}\text { Calcineurin inhibitor immunosuppressant, folate analog metabolic inhibitor, } \\
\text { purine antimetabolite }\end{array}$ \\
\hline $\begin{array}{l}\text { Nonopioid cough } \\
\text { suppressants } \\
\text { and expectorants }\end{array}$ & & & $\checkmark$ & & & & $\begin{array}{l}\text { Expectorant, non-narcotic antitussive, } \sigma-1 \text { agonist, uncompetitive } \\
\text { N-methyl-D-aspartate receptor antagonist }\end{array}$ \\
\hline Antimicrobials & & & $\checkmark$ & $\checkmark$ & & & $\begin{array}{l}\text { Macrolide, cephalosporin, penicillin class, rifamycin, non-nucleoside analog reverse } \\
\text { transcriptase inhibitor, influenza A M2 protein inhibitor }\end{array}$ \\
\hline Others & $\checkmark$ & $\sqrt{ }$ & $\checkmark$ & $\checkmark$ & $\checkmark$ & $\checkmark$ & $\begin{array}{l}\beta \text {-3-adrenergic agonist, methylxanthine, cholinesterase inhibitor, interferon } \alpha \text { and } \beta \text {, } \\
\text { partial cholinergic nicotinic agonist, tyrosine hydroxylase, retinoid, serotonin-1b } \\
\text { and serotonin-1d receptor agonist, stimulant laxative, vitamin K antagonist, } \\
\text { platelet aggregation inhibitor }\end{array}$ \\
\hline
\end{tabular}

NOTE: Associated syndrome checked if at least 2 or more medications within the wider class are associated with the syndrome. Abbreviations: NSAIDs, nonsteroidal anti-inflammatory drugs.

relevant references for their inclusion is presented in the Supporting Information, Appendix 1, in the online version of this article. Table 1 summarizes these medications across 18 major drug categories. Antiepileptics were linked to all 6 geriatric syndromes, whereas antipsychotics, antidepressants, antiparkinsonism, and opioid agonists were associated with 5 syndromes. Ten of the 18 categories were associated with 3 geriatric syndromes-cognitive impairment, delirium, and falls. Four medication categories were associated with only 1 syndrome. Nonopioid/nonsteroidal antiinflammatory and/or analgesics and nonopioid cough suppressant and expectorant medications were associated with falls syndrome only. Hormone replacement medications were associated with depression only, and immunosuppressants were associated with unintentional weight and appetite loss only.
Approximately $58 \%$ of the medications overlapped with the AGS 2015 Beer's Criteria ${ }^{20}$ irrespective of whether the specific syndrome association was stated in the rationale. ${ }^{20}$ Medications that overlapped were mostly in the delirium, cognitive impairment, and falls category with only a few overlaps in depression, unintentional weight loss, and urinary incontinence lists (see Supporting Information, Appendix 1, in the online version of this article).

\section{Phase 2: Prevalence of MAGS}

Among 154 participants, the mean age was 76.5 $( \pm 10.6)$ years, $64.3 \%$ were female, $77.9 \%$ were white, and $96.1 \%$ non-Hispanic. The median hospital length of stay was 6 days, with an interquartile range of 5 days. The orthopedic service discharged the highest proportion of patients $(24 \%)$, followed by the 
geriatrics and internal medicine services, which each discharged $19.5 \%$ of the patients (Table 2). The remaining participants of the larger qualityimprovement project $(\mathrm{N}=939)$ did not significantly differ on these demographic and clinical characteristics except for hospital length of stay, which was shorter in the sample analyzed (see Supporting Information, Appendix 2, in the online version of this article).

Patients were discharged to SNFs with an average of $14.0( \pm 4.7)$ medication orders. Overall, $43 \%$ $( \pm 13 \%)$ of these discharge medication orders were MAGS. Every patient in the sample was ordered at least 1 medication associated with geriatric syndromes. Multiple MAGS were the norm, with an average of $5.9( \pm 2.2)$ MAGS per patient. MAGS were also the norm, as $98.1 \%$ of the sample had medica-

\begin{tabular}{|c|c|}
\hline Baseline Characteristics & Mean $( \pm$ SD) or Percent $(n)$ \\
\hline Age, $y$ & $76.5( \pm 10.6)$ \\
\hline \multicolumn{2}{|r|}{$10.01-90.0)$} \\
\hline Female & $64.3 \%(99)$ \\
\hline \multicolumn{2}{|l|}{ Race } \\
\hline White & $77.9 \%(126)$ \\
\hline Black & $16.2 \%(25)$ \\
\hline Unknown & $0.6 \%(1)$ \\
\hline Declined & $0.6 \%(1)$ \\
\hline Missing & $0.6 \%(1)$ \\
\hline \multicolumn{2}{|l|}{ Ethnicity } \\
\hline Non-Hispanic & $96.1 \%(148)$ \\
\hline Hispanic & $1.3 \%(2)$ \\
\hline Unknown & $2.6 \%(4)$ \\
\hline Hospital length of stay, d & $7.0( \pm 4.2)$ \\
\hline Hospital length of stay, d, median (IQR) & $6.0(5.0)$ \\
\hline №. of hospital discharge medications, count & $14.0( \pm 4.7)$ \\
\hline \multicolumn{2}{|l|}{ Discharge service } \\
\hline Orthopedic service & $24.0 \%(37)$ \\
\hline Geriatric service & $19.5 \%(30)$ \\
\hline Internal medicine & $19.5 \%(30)$ \\
\hline Other & $37.0 \%(57)$ \\
\hline
\end{tabular}

NOTE: Abbreviations: IQR, interquartile range; SD, standard deviation. tion orders associated with at least 2 different syndromes.

When the Beer's criteria ${ }^{20}$ were applied to the medication orders (instead of the MAGS list), problematic medications appeared less common. Patients had an average of 3.04 ( \pm 1.7$)$ MAGS that were also listed on the AGS 2015 Beer's list, ${ }^{20}$ representing an average of $22.3 \%$ of all discharge orders.

Table 3 illustrates the average number of medications per patient associated with each syndrome, and the percentage of patients (number in parentheses) discharged with at least 1 medication associated with each syndrome per the MAGS list and the Beers 2015 criteria. $^{20}$ For example, per the MAGS list, the syndrome most frequently associated with medications was falls, with patients discharged on an average of $5.5( \pm 2.2)$ medications associated with falls, and $100 \%$ of the sample had at least 1 discharge medication associated with falls. Alternatively, the syndrome associated with the lowest frequency of medications was unintentional weight loss (with an average of 0.38 medications per patient), although $36 \%$ of these patients had more than 1 discharge medication associated with weight loss. As seen in Table 3, the mean and prevalence of 1 or more medications associated with each of the geriatric syndromes as identified by the Beers 2015 criteria $^{20}$ was lower than those identified by the MAGS list developed for this study.

\section{DISCUSSION}

An iterative process of evidence review by a multidisciplinary panel resulted in a list of 513 medications associated with 6 common geriatric syndromes. This analysis demonstrated that hospitalized, older patients discharged to SNFs were frequently prescribed MAGS. The rate of prescribing ranged from $100 \%$ of patients with a medication associated with falls to $36 \%$ for unintentional weight loss. Moreover, an alarming $43 \%$ of all medications at hospital discharge were MAGS. For this vulnerable population, the combination of high prevalence of MAGS and high risk of geriatric syndromes emphasize a need to critically

TABLE 3. Prevalence of Medications Associated With Geriatric Syndromes per MAGS and AGS Beers 2015 Criteria in an Older Cohort of Hospitalized Patients Discharged to Skilled Nursing Facilities ( $N=154)$

\begin{tabular}{lcccc}
\hline & \multicolumn{2}{c}{ Associated Medications per MAGS List } & & \multicolumn{2}{c}{ Associated Medications per AGS Beers 2015 Criteria } \\
\cline { 2 - 4 } Geriatric Syndromes & Mean \pm SD & $\begin{array}{c}\text { Percentage of Patients Receiving } \\
\geq 1 \text { Related Medication }\end{array}$ & & $\begin{array}{c}\text { Percentage of Patients Receiving } \\
\geq 1 \text { Related Medication }\end{array}$ \\
\hline Cognitive impairment & $1.8 \pm 1.2)$ & $84.4 \%(130)$ & $1.6( \pm 1.2)$ & $78.6 \%(121)$ \\
Delirium & $1.4 \pm 1.1)$ & $76.0 \%(117)$ & $1.3 \pm 1.2)$ & $68.2 \%(105)$ \\
Falls & $5.5( \pm 2.2)$ & $100 \%(154)$ & $2.6( \pm 1.6)$ & $92.2 \%(142)$ \\
Unintentional weight and/or appetite loss & $0.4 \pm 0.5)$ & $36.3 \%(56)$ & $0.1 \pm 0.3)$ & $6.5 \%(10)$ \\
Urinary incontinence & $1.6 \pm 1.0)$ & $85.7 \%(132)$ & $0.1 \pm 0.2)$ & $5.8 \%(9)$ \\
Depression & $1.7 \pm 1.0)$ & $90.9 \%(140)$ & $0.0( \pm 0.0)$ & $0.0 \%(0)$ \\
All syndromes & $5.9 \pm 2.2)$ & $100 \%(154)$ & $3.0( \pm 1.7)$ & $95 \%(149)$ \\
\hline
\end{tabular}

NOTE: Abbreviations: AGS, American Geriatric Society; MAGS, Medications Associated With Geriatric Syndromes, SD, standard deviation. 
review the risks and benefits of MAGS throughout hospitalization and at the time of discharge.

A body of evidence demonstrates that many drugs in a typical older adult regimen have no specific clinical indication, are considered inappropriate, or have uncertain efficacy in the geriatric population. ${ }^{24-26}$ This study builds on the foundational work described in landmark reviews such as the AGS Beers ${ }^{20}$ and STOPP/START ${ }^{21}$ (Screening Tool of Older Persons' Potentially Inappropriate Prescriptions/Screening Tool to Alert doctors to Right, i.e. appropriate indicated Treatment) criteria. Both of these tools, however, were specifically designed as screening tools to identify medications considered unsafe for older adults under most circumstances and within specific illness states. ${ }^{19-21}$ They are most often utilized when starting a medication to avoid acute adverse events. In contrast, the MAGS list was developed to be inclusive of medications that are often appropriate for many medical diagnoses but may also contribute to underlying geriatric syndromes that are more chronic in nature. In addition, inclusion of such medicines increases the sensitivity of screening for medications that can be targeted through patient-centered deprescribing efforts when clinically appropriate.

A major strength of this study is that we bring together evidence across a spectrum of geriatric syndromes commonly experienced by hospitalized elders. In addition to evaluating multiple syndromes, we applied multiple modalities; particularly the use of an iterative review process by a multidisciplinary team of experts and using Lexicomp and FDA insert packages for linking medications to specific geriatric conditions. The inclusion criteria were broadened beyond single sources of evidence in an effort to capture a comprehensive list of medications. As a result, the MAGS list can be implemented as a screening tool for deprescribing interventions and assessing medication appropriateness to address individual or clusters of geriatric syndromes within a patient.

In addition to expanding this knowledge base, clinical relevance of the MAGS list is highlighted by its application to a sample of hospitalized older adults discharged to SNFs, a cohort known to experience geriatric syndromes. In fact, $43 \%$ of patients' medications at hospital discharge were MAGS. Importantly, due to the cross-sectional nature of this study, we cannot be certain if the medication caused or potentiated each of the geriatric syndromes. However, hospitals and SNFs are devoting major resources toward reduction of falls, avoidance of urinary catheter use, and reduction of preventable readmissions. These efforts can be complemented by considering the number of medications associated with falls, urinary incontinence, and overall MAGS burden. The striking prevalence of MAGS demonstrates a rigorous need to weigh the risks and benefits of these medications. Above all, the intent of this study is not to propose that any MAGS be reflexively stopped, but rather that the MAGS list should facilitate a holistic approach to care for the complex older adult. For example, standard therapies such as gabapentin may be appropriate for treating neuralgic pain but may also contribute to falls and urinary incontinence. Thus, alternative pain treatments could be selected in place of gabapentin for a 75-year old patient who is experiencing recurrent falls and increasing incontinence. Therefore, the MAGS list enables a patient-provider discussion wherein medications' therapeutic benefits can be weighed against risks posed by specific clusters of geriatric syndromes, potential impact on quality of life, and consistency with goals of care.

This study has some limitations. First, although we examined a broad number of geriatric syndromes, several other geriatric syndromes experienced by hospitalized older adults were not addressed including: fecal incontinence, insomnia, and functional impairment. These syndromes were intentionally excluded from the study a priori due to reasons of feasibility and scope. Second, unlike the Beer's 2015 criteria, the MAGS list does not sub-classify associations of medications with geriatric syndromes for patients with specific diseases (eg, heart failure). In fact, our MAGS list included medications often indicated in treating these diagnoses. A clinician must work with the patient to weigh the disease-specific benefits of some medications with the potential effect on geriatric syndrome symptoms and outcomes. Third, the instrument has a very high sensitivity, which was intended to generate an inclusive list of medications that enables providers to weigh risks of geriatric syndromes with the intended indication benefit. The objective is not to use this list as a reflexive tool but rather help clinicians identify a starting point to address geriatric syndromes in their patients to make patient-centered medication decisions. Although the MAGS list is intentionally large (sensitive), the advent of advanced bioinformatics can enable MAGS to be assessed in the future for both clinical and research purposes. Fourth, FDA insert packages and Lexicomp databases report anything experienced by the patient while on the particular medication, but it might not necessarily imply a causative link. The high use of MAGS and the specific geriatric syndrome may coexist due to the high prevalence and interplay of multimorbidity, polypharmacy, and geriatric syndromes in this population. Last, the list was developed by expert panel members predominantly from a single institution, which may introduce bias. Despite these limitations, the prevalence of these medications in a sample of patients transitioning from acute to post-acute care highlights the utility of the MAGS list in future clinical research and quality improvement endeavors.

In conclusion, the MAGS list provides a comprehensive and sensitive indicator of medications associated with any of 6 geriatric syndromes regardless of 
medication indication and appropriateness. The MAGS list provides an overall degree of medication burden with respect to geriatric syndromes and a foundation for future research to assess the relationship between the presence of geriatric syndromes and syndromeassociated medications. The MAGS list is an important first step in summarizing the data that link medications to geriatric syndromes. Future studies are needed to broaden the analysis of MAGS for other common geriatric syndromes and to identify new and emerging medications not present during the time of this analysis. The MAGS list has the potential to facilitate deprescribing efforts needed to combat the epidemic of overprescribing that may be contributing to the burden of geriatric syndromes among older patients.

\section{Acknowledgements}

The authors thank Dr. Linda Beuscher, Dr. Patricia Blair Miller, Dr. Joseph Ouslander, Dr. William Stuart Reynolds, and Dr. Warren Taylor for providing their expertise and participating in the expert panel discussions that facilitated the development of the MAGS list. The authors also recognize the research support provided by Christopher Simon Coelho.

Disclosures: This research was supported by the Department of Health and Human Services, Centers for Medicare \& Medicaid Services grant \#1C1CMS331006 awarded to Principal Investigator, John F. Schnelle, $\mathrm{PhD}$. Dr. Vasilevskis was supported by the National Institute on Aging of the National Institutes of Health award K23AG040157 and the Geriatric Research, Education and Clinical Center. Dr. Bell was supported by National Institute on Aging-K award K23AG048347-01A1. Dr. Mixon is supported by a Veterans Affairs Health Services Research \& Development Career Development Award (12-168). This research was also supported by the National Center for Advancing Translational Sciences Clinical and Translational Science award UL1TR000445. The contents of this publication are solely the responsibility of the authors and do not necessarily represent the official views of the US Department of Health and Human Services or any of its agencies, the National Center for Advancing Translation Science, the National Institutes of Health, or the Department of Veterans Affairs. Each coauthor contributed significantly to the manuscript. Dr. Kripalani has received stock/stock options from Bioscape Digital, LLC. None of the other authors have significant conflicts of interest to report related to this project or the results reported within this article.

\section{References}

1. Inouye SK, Studenski S, Tinetti ME, Kuchel GA. Geriatric syndromes: clinical, research, and policy implications of a core geriatric concept. J Am Geriatr Soc. 2007;55:780-791.

2. Tinetti ME, Inouye SK, Gill TM, Doucette JT. Shared risk factors for falls, incontinence, and functional dependence. Unifying the approach to geriatric syndromes. JAMA. 1995;273:1348-1353.

3. Olde Rikkert MG, Rigaud AS, van Hoeyweghen RJ, de Graaf J. Geriatric syndromes: medical misnomer or progress in geriatrics? Neth J Med. 2003;61:83-87.

4. Buurman BM, Hoogerduijn JG, de Haan RJ, et al. Geriatric conditions in acutely hospitalized older patients: prevalence and one-year survival and functional decline. PLoS One. 2011;6:e26951.
5. Wang HH, Sheu JT, Shyu YI, Chang HY, Li CL. Geriatric conditions as predictors of increased number of hospital admissions and hospital bed days over one year: findings of a nationwide cohort of older adults from Taiwan. Arch Gerontol Geriatr. 2014;59:169-174.

6. Cigolle CT, Langa KM, Kabeto MU, Tian Z, Blaum CS. Geriatric conditions and disability: the Health and Retirement Study. Ann Intern Med. 2007;147:156-164.

7. Lakhan P, Jones M, Wilson A, Courtney M, Hirdes J, Gray LC. A prospective cohort study of geriatric syndromes among older medical patients admitted to acute care hospitals. J Am Geriatr Soc. 2011;59: 2001-2008.

8. Bell SP, Vasilevskis EE, Saraf AA, et al. Geriatric syndromes in hospitalized older adults discharged to skilled nursing facilities. J Am Geriatr Soc. 2016;64(4):715-722.

9. Allen LA, Hernandez AF, Peterson ED, et al. Discharge to a skilled nursing facility and subsequent clinical outcomes among older patients hospitalized for heart failure. Circ Heart Fail. 2011;4:293-300.

10. Creditor MC. Hazards of hospitalization of the elderly. Ann Intern Med. 1993;118:219-223.

11. Flood KL, Rohlfing A, Le CV, Carr DB, Rich MW. Geriatric syndromes in elderly patients admitted to an inpatient cardiology ward. J Hosp Med. 2007;2:394-400.

12. Lund BC, Schroeder MC, Middendorff G, Brooks JM. Effect of hospitalization on inappropriate prescribing in elderly Medicare beneficiaries. J Am Geriatr Soc. 2015;63:699-707.

13. Gnjidic D, Hilmer SN, Blyth FM, et al. Polypharmacy cutoff and outcomes: five or more medicines were used to identify communitydwelling older men at risk of different adverse outcomes. J Clin Epidemiol. 2012;65:989-995.

14. Best O, Gnjidic D, Hilmer SN, Naganathan V, McLachlan AJ. Investigating polypharmacy and drug burden index in hospitalised older people. Intern Med J. 2013;43:912-918.

15. Hines LE, Murphy JE. Potentially harmful drug-drug interactions in the elderly: a review. Am J Geriatr Pharmacother. 2011;9:364-377.

16. Dechanont S, Maphanta S, Butthum B, Kongkaew C. Hospital admissions/visits associated with drug-drug interactions: a systematic review and meta-analysis. Pharmacoepidemiol Drug Saf. 2014;23:489-497.

17. Rochon PA, Gurwitz JH. Optimising drug treatment for elderly people: the prescribing cascade. BMJ. 1997;315:1096-1099.

18. Wierenga PC, Buurman BM, Parlevliet JL, et al. Association between acute geriatric syndromes and medication-related hospital admissions. Drugs Aging. 2012;29:691-699.

19. American Geriatrics Society Beers Criteria Update Expert P. American Geriatrics Society updated Beers Criteria for potentially inappropriate medication use in older adults. J Am Geriatr Soc. 2012;60:616-631.

20. By the American Geriatrics Society 2015 Beers Criteria Update Expert Panel. American Geriatrics Society 2015 updated Beers criteria for potentially inappropriate medication use in older adults. J Am Geriatr Soc. 2015;63:2227-2246.

21. Gallagher P, O'Mahony D. STOPP (Screening Tool of Older Persons' potentially inappropriate Prescriptions): application to acutely ill elderly patients and comparison with Beers' criteria. Age Ageing. 2008;37:673-679.

22. Mant J, Hobbs FDR, Fletcher K, et al. Warfarin versus aspirin for stroke prevention in an elderly community population with atrial fibrillation (the Birmingham Atrial Fibrillation Treatment of the Aged Study, BAFTA): a randomised controlled trial. Lancet. 2007;370:493-503.

23. U.S. Food and Drug Administration. Drugs. Available at: http://www. fda.gov/Drugs/default.htm. Accessed May 15th, 2015.

24. Hanlon JT, Artz MB, Pieper CF, et al. Inappropriate medication use among frail elderly inpatients. Ann Pharmacother. 2004;38:9-14.

25. Morandi A, Vasilevskis EE, Pandharipande PP, et al. Inappropriate medications in elderly ICU survivors: where to intervene? Arch Intern Med. 2011;171:1032-1034.

26. Schmader K, Hanlon JT, Weinberger M, et al. Appropriateness of medication prescribing in ambulatory elderly patients. J Am Geriatr Soc. 1994;42:1241-1247. 